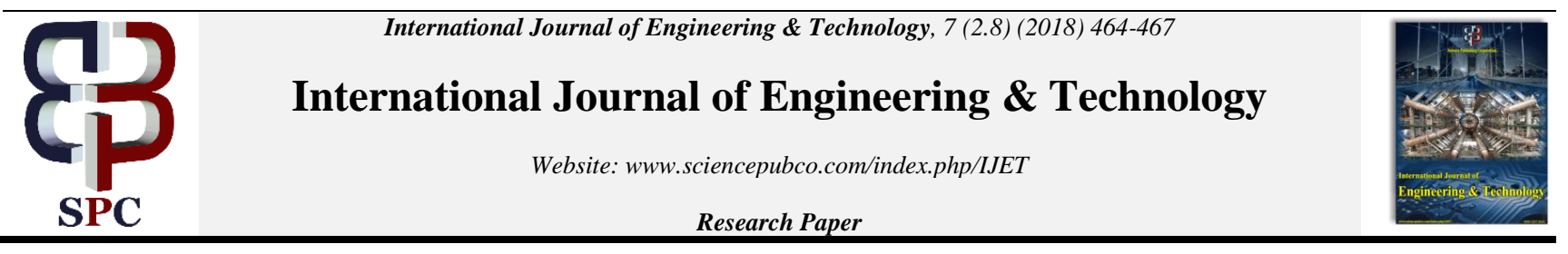

\title{
Survey on short-term load forecasting using hybrid neural network techniques
}

\author{
Shaive Dalela ${ }^{1}$, Aditya Verma ${ }^{2}$, A.L. Amutha ${ }^{3}$ \\ ${ }^{1,2} U G$ Student, ${ }^{3}$ Assistant Professor $(O G)$ \\ ${ }^{1,2,3}$ Computer Science and Engineering, SRM University, Chennai, India
}

\begin{abstract}
Load forecasting is an issue of great importance for the reliable operation of the electric power system grids. Various forecasting methodologies have been proposed in the international research bibliography, following different models and mathematical approaches. In the current work, several latest methodologies based on artificial neural networks along with other techniques have be discussed, in order to obtain short-term load forecasting. In this paper, approaches taken by different researchers considering different parameters in means of predicting the lease error has been shown. The paper investigates the application of artificial neural networks (ANN) with fuzzy logic (FL), Genetic Algorithm(GA), Particle Swarm Optimization(PSO) and Support Vector Machines(SVM) as forecasting tools for predicting the load demand in short term category. The extracted outcomes indicate the effectiveness of the proposed method, reducing the relative error between real and theoretical data.
\end{abstract}

Keywords: Short-term load forecasting, Artificial Neural Network (ANN), Fuzzy Logic (FL), Genetic Algorithm (GA), Support Vector Machine (SVM), Particle Swarm Optimization (PSO).

\section{Introduction}

Load forecasting requires staunch operation and cost-effective utilization of power system and its resources which can vary from few minutes to several days. The forecasting of hourly - integrated loads carried out for one day to one week ahead is usually referred to as short-term load forecasting (STLF). Short-Term Load Forecasting (STLF) predicts system load by using historic load and weather data, plus forecasted weather conditions. Since system load usually differs significantly on certain days of the year, the program uniquely classifies such days to allow separate analyses of them. Short-Term Load Forecasting can be performed for a power system consisting of a single load area or multiple load areas. For a system of multiple load areas, Short-Term Load Forecasting can be installed with multiple copies for the individual load areas in the system. The forecast results can be transferred to spreadsheets for performing various data analyses. Load forecasting helps the operator managing the systemto schedule demand allocation in a proficient manner. In addition, load forecasting can also help in providing power produced exchange with other services. In addition to these economic reasons, load forecasting is also useful for system security.

Traditional computing approaches, such as regression and interpolation, certainly cannot provide the results that are required. On the other hand, complex algorithmic methods involving lots of calculations can converge slowly and may diverge in certain cases. Various algorithms have been put forward to improve the accuracy of the forecasting problem [1]. According to the study carried out in load forecasting it can be generally classified into two categories in accordance with the techniques. One approach takes the load pattern and determines its relation with time and predicts the future load by using various time series analysis techniques [2-8]. Other approaches focus the load pattern dependence on the weather variables, and establishes a relationship between the weather variables and the system loads. The future load is then predicted by variating weather information along with present historical data to present a relation among them [9-12].

\section{Some Definitions}

\section{A. Load Forecasting}

Load forecasting is a procedure used by energy operating companies to predict the energy needed to meet the demand and supply balance in order to maintain sustainable usage. The accuracy of forecasting is of great connotationfor the functional and controlledloading of a service company. It helps in saving a lot of money for the enterprise applying the same.

\section{B. Fuzzy Logic}

Fuzzy logic is an approachin computing of many-valued logic in which the degree of truth values may be any real number between 0 and 1. It is carriedout to manage the concept of partial truth, where the variables may range between complete truth and complete false.

\section{Artificial Neural Network}

Artificial neural networks (ANNs) or connectionist systems are computing systems inspired based on the structure and functions of biological neural networks. Such systems learn by dynamically improving performance on tasks by considering examples. They do not rely on task-bounded programming.

\section{Backpropagation Algorithm}


Backpropagation is a method used in artificial neural networks to calculate a gradient that is needed in the calculation of the weights to be used in the network.

\section{E. Support Vector Machine}

In machine learning, support vector machines (SVMs, also support vector networks) are supervised learning models which can be used for both classification and regression challenges. It is a point nearer to the hyper-plane that separates two(or more) classes.

\section{F. Particle Swarm Optimization}

In computer science, particle swarm optimization (PSO) is a computational method that improves the solution to a problem by continuously trying to improve a candidate solution in respect to a given measure of quality.

\section{Practical Application (Existing)}

Recent hybrid training methods for ANN for short term load forecasting can be classified as follows:

I) ANN with Fuzzy Logic

II) ANN with Support Vector Machine

III) ANN with Particle Swarm Optimization

IV) ANN with Genetic Algorithm

\section{1) ANN with Fuzzy Logic}

A fuzzy logic load forecasting ANN model is generally developed to classify a large input load data set to predict the load demand. Senjyu, T.; Mandai, P.; Uezato, K.; Funabashi, T., "Next day load curve forecasting using hybrid correction method,"(2005) [13].This paper suggests a methodology for short-term load forecasting, based on hybrid correction method. Standard neural network based short-term load forecasting techniques have shortcomings especially when weather causes changes in season. Hence, they proposed a load correction method by using an approach in which a fuzzy logic, based on historical data corrects the neural network output to obtain the next day forecasted load. A Euclidean norm is used for the selection of similar days along with weighted factors. The load correction method for the generation of new similar days is also proposed.

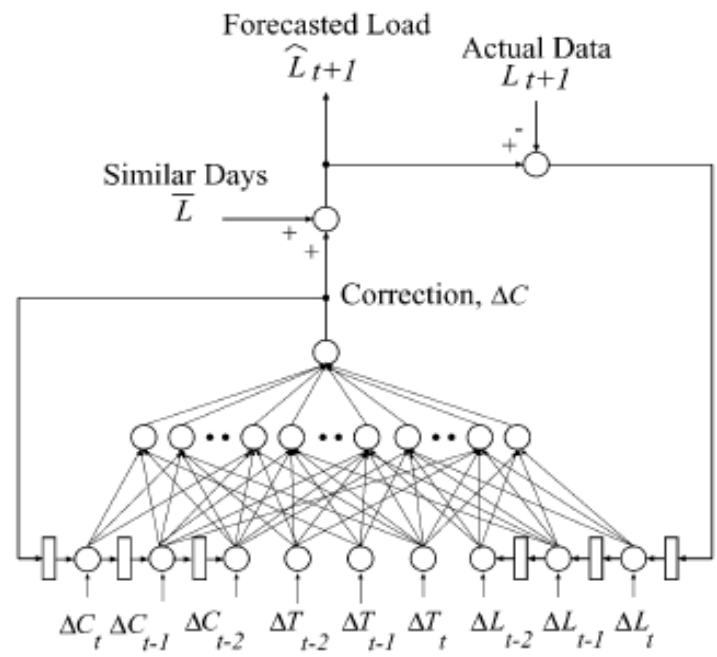

Fig. 1: Neural Network along with Fuzzy Logic for output correction

It is also helpful in figuring out the discomfort level of the given day forecasts by the following formula

$$
D I_{t+1}=0.81 T_{t+1}+0.01 U_{t+1}\left(0.99 T_{t+1}-14.3\right)+46.3
$$

Where, $D I_{t+1}$ is discomfort index, is forecasted temperature $T_{t+1}\left[{ }^{\circ} \mathrm{C}\right]$, and is forecasted relative humidity $U_{t+1}[\%]$ at hour The range of discomfort is also specified as follows -

$$
\text { Comfort: } \mathrm{DI} \leq 72
$$

Slightly discomfort: $72<$ DI $<76$

Discomfort: DI $\geq 76$

The above has some accumulative effect which forces the customers to keep the same load nature for coming days to come unless some drastic change in environment takes place.

\section{2) ANN with Support Vector Machine}

Recently the reported research using support vector machine and more on support vector regression were introduced for STLF.

Afshin, M.; Sadeghi an, A, "PCA-based Least Squares Support Vector Machines in Week-Ahead Load Forecasting,"(2007) [14].This paper lays down the operation of principal component analysis (PCA) to least squares support vector machines (LSSVM) in a forecasting problem with output of load which is of a week ahead. New practical features are added for better and more potenttraining of the model. For instance, it has been found out that hours of daylight is a prominent factor in shaping the load profile. In case of cities that are situated in the northern hemisphere, this is a major consideration.Analysis proves that the feature extraction also plays a major role in accurate results. Steps for carrying out this process is given as follows -

Step 1: Abnormal samples present in the dataset are removed by preprocessing. The data is then normalized to zero mean and unit variance.

Step 2: By trial and error, number of features that are to be entered into the LS-SVM model (minimum fraction variance component) are determined after the implementation of PCA on the input data. Step 3: Prediction of the next seven day's maximum load demands using the test data sets can be carried out by the following formula

$-y(x)=\sum_{i=1}^{N} \alpha_{i} K\left(x_{i}, x_{j}\right)+b$

Some parameters have to be selected which influence the performance of the model when training a LS-SVM model considerably:

* Regularization parameter $(\gamma)$

* Kernel bandwidth parameter $(\delta)$

* The kernel function, $K\left(x_{i}, x_{j}\right), \mathrm{RBF}$ is used in this LSSVM model.

* The size of training data sets, i.e. number of previous days that are included for one training data.

Step 4: Bayesian evidence framework is applied to theLS-SVM regression algorithm and estimation is performed on the optimal regularization parameter $(\gamma)$ and kernel parameter $(\delta)$ until accuracy reached is enough to carry out the task efficiently.

\section{3) ANN with Particle Swarm Optimization}

Particle swarm optimization is a population-based algorithm, which is widely applied to the optimization problems in which simple software agents move in search space to provide best possible result. Various researches concerned with it have been implemented, different hybrid and updated versions of PSO are available, e.g., QPSO, MPSO, and the adaptive PSO, which shows better training results of neural networks.

Ning Lu; Jianzhong Zhou, "Particle Swarm Optimization-Based RBFNeural Network Load Forecasting Model,"(2009) [15]. In this paper, improvement in the precision of electric power system for short term load forecasting has been proposed through a new model.Both Particle Swarm Optimization (PSO) algorithm and radial basis function (RBF) neural network are taken into use in this paper.For optimizing the weighting factor of neural network PSO is applied. The load forecasting model which is optimized by PSO has more accuracy than the conventional RBF Neural Network 
model according to the theoretical analysis and simulations. It also shows the importance of the two primary operators - velocity update and position update which help in minimizing error.

Step 1: Parameters of RBF network are initialized.

Subtractive clustering algorithm determinesthe center vector $\mathrm{C}$ and the width parameter $\sigma$. Optimization of the inertia weighting factor is carried out by PSO. Initialize the inertia weighting factor $w_{j, i}$ $=1\left(0 \leq w_{j, i} \leq 1\right)$.

PSO algorithm parameters are initialized. Set $\mathrm{c} 1=1, \mathrm{c} 2=1, \mathrm{~T}=1$, $T_{\max }=10000$, and $\mathrm{r} 1, \mathrm{r} 2$ are two random number.

Step 2: The sample data is normalized with the equation as:

$X_{t}=\frac{X_{t}-X_{\min }}{X_{\max }-X_{\min }} \mathrm{t}=(1,2 \ldots 24)$

Where $X_{t}$ is the load data of hour t,the maximum of the load sample data is $X_{\text {max }}$ andis the minimum of the load sample datais $X_{\min }$. Step 3: Fitness function is defined as follow:

$$
E=\sum_{j=1}^{n}\left[\sum_{k=1}^{n}\left(t_{j k}-y_{j k}\right) \wedge 2\right] / n
$$

Where, target value is $t_{j k}$; potential output value is $y_{j k}$; the nodes of output is $\mathrm{m}$, the numbers of training data is $\mathrm{n}$. According to the fitness, if this current position $P_{i}$, better than the position $P_{\text {best }, i}$, then it will take its place.Otherwise, $P_{b e s t, i}$ remains unchanged. Step 3: The minimum of $P_{\text {best }, i}$ is chosen and previous $P_{q, b e s t}$ value is compared with it, if less than the previous one, then take the place of the previous $P_{q, \text { best }}$.

Step 4: Let $\mathrm{T}=\mathrm{T}+1$

Step 5: The condition for stopping the iteration is accessed. The iteration is halted if the maximal iterative times are satisfied and $\mathrm{E}<\varepsilon$ ( $\varepsilon$ is a given parameter) and $P_{q, \text { best }}$ is the best solution which represents the weighting factor $w_{i j}$. Otherwise, the velocity and new position of each particle is calculated in accordance toequation (1) and equation (2) and the steps are repeated from step2.

Step 6: In accordance to the optimized parameter $w_{i j}$, to forecast the load data for wantedRBF network structure has been established.

\section{4) ANN with Genetic Algorithm}

The genetic algorithm (GA) is a random search technique inspired by the process of natural selectionthat is widely used to find the optimal solution. Optimal point in the populations helps in determination of the optimal solution of the problem under consideration. The fitness value helps in the finding the next offspring by carrying out the process.

This paper presents a methodology which optimizes by improving the accuracy of predictions by back propagation neural network based genetic algorithm (GA).The weight upgradation is attained by selection, crossing and mutation operations with GA's optimizing and BP neural network's dynamic featuring. The performance of BP neural network based genetic algorithm optimization predictions are compared with that of BP network using load time series from a practical power system.

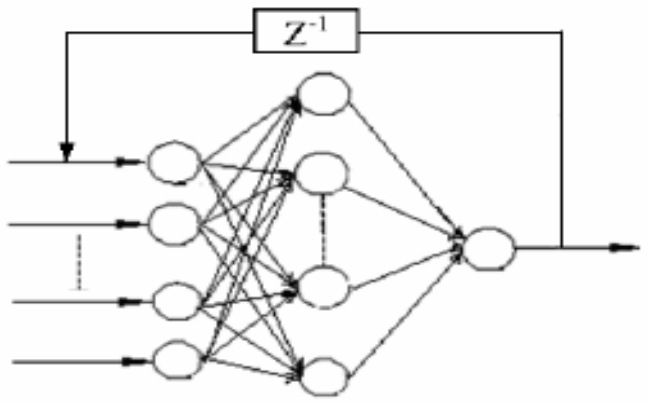

Fig. 2: Neural Network improved by Genetic Algorithm

$$
\begin{aligned}
d\left(X_{i}\right) & = \begin{cases}\left(b_{i}-X_{i}\right)[r(1-t)]^{b} & \text { if sign }=0 \\
\left(\mathrm{X}_{\mathrm{i}}-\mathrm{a}_{\mathrm{i}}\right)[r(1-t)]^{b} & \text { if } \text { sign }=1\end{cases} \\
X_{i}^{\prime} & = \begin{cases}X_{i}+d\left(X_{i}\right) & \text { if sign }=0 \\
X_{i}-d\left(X_{i}\right) & \text { if sign }=1\end{cases}
\end{aligned}
$$

According to the neural network's weight and threshold of correspondence, the quadratic sum of error is calculated.

$$
\begin{gathered}
E\left(X_{i}\right)=\frac{1}{2 n} \sum_{p=1}^{n} \sum_{q=1}^{c}\left(d_{p q}-o_{p q}^{i}\right)^{2} \\
f(x)=\frac{1}{1+E}
\end{gathered}
$$

\section{Conclusion}

Load forecasting plays an important role in providing the positiveworking of a power system. Perhaps, it could also provide benefits in liberalization of the electricity market. This paper evaluates the recent published work to put focus on hybrid neural network model as a possible answer for the short-term load forecasting problem. All the recent models of some hybrid NN techniques are mentioned in this paper. Consideration of all the environmental factors has made the load forecasting more accurate in comparison to the normal technique models.Many improvements can still be made in the current models that have been put forward in the past.

\section{References}

[1] M. Q. Raza*, Z. Baharudin, "A Review on Short Term Load Forecasting Using Hybrid Neural Network Techniques" in proceedings of 2012 IEEE International Conference on Power and Energy (PECon), 2-5 December 2012

[2] C. Ying, et aI., "Short-Term Load Forecasting: Similar DayBased Wavelet Neural Networks," Power Systems, IEEE Transactions on, vol. 25,pp. 322-330,2010.

[3] S. J. Huang and K. R. Shih, "Short-term load forecasting via ARMAmodel identification including non-Gaussian process considerations,"IEEE Trans. Power Syst., vol. 18, no. 2, pp. 673 679, 2003.

[4] A. Khosravi, et aI., "Construction of Optimal Prediction Intervals for Load Forecasting Problems," Power Systems, IEEE Transactions on, vol. 25, pp. 1496-1503,2010

[5] S. Rahman and O. Hazim, "A generalized knowledge-based short-term load-forecasting technique," IEEE Trans. Power Syst. , vol. 8, no. 2, pp.508-514,1993.

[6] J. W. Taylor and R. Buizza, "Neural network load forecasting with weather ensemble predictions," IEEE Trans. Power Syst., vol. 17 , no. 3 , pp. 626-632, 2002.

[7] Mohammed EI-Telbany, Fawwaz EI-Karmi, Short-term forecasting of Jordanian electricity demand using particle swarm optimization, Electric Power Systems Research, Volume 78, Issue 3, March 2008, Pages 425-433, ISSN 0378-7796, IO.IO I6/j.epsr.2007.03.0 II.

[8] T. W. S. Chow and C. T. Leung, "Neural networks based shortterm load forecasting using weather compensation," IEEE Trans. Power Syst., vol. I I, no. 4, pp. 1736-1742, Nov. 1996.

[9] Osman, Z.H.; Awad, M.L.; Mahmoud, T.K.; , "Neural network based approach for short-term load forecasting," Power Systems Conference and Exposition, 2009. PSCE '09. IEEE/PES, vol., no., pp.I-8, 15-18 March 2009.

[10] Tasre, M.B.; Bedekar, P.P.; Ghate, V.N.; , "Daily peak load forecasting using ANN," Engineering (NUiCONE), 2011 Nirma University International Conference on , vol., no., pp.I-6, 8-10 Dec. 2011.

[11] De Felice, M.; Xin Yao;, "Short-Term Load Forecasting with Neural Network Ensembles: A Comparative Study [ApplicationNotes]," Computational Intelligence Magazine, IEEE , vol.6, no.3, pp.47-56, Aug. 2011

[12] D. W. Bunn and E. D. Farmer, Comparative Models for Electrical LoadForecasting .. Chichester, U.K.: Wiley, 1985. 
[13] [13] Senjyu, T.; Mandai, P.; Uezato, K.; Funabashi, T.; , "Next day load curve forecasting using hybrid correction method," Power Systems, IEEE Transactions on , vo1.20, no.1, pp. 102109, Feb. 2005.

[14] Li, G., Cheng, c.-I., Lin, J.-Y., Zeng, Y., Short-term load forecasting using support vector machine with SCE-UA algorithm. In: Third International Conference on Natural Computation (ICNC 2007). IEEE, pp. 290-294

[15] Ning Lu; Jianzhong Zhou; , "Particle Swarm Optimization-Based RBF Neural Network Load Forecasting Model," Power and Energy Engineering Conference, APPEEC 2009.

[16] Yan Wang, Yuanwei Jing, WeilunZhao,Yan-e Mao, "Dynamic Neural Network Based Genetic Algorithm Optimizing for Short Term Load Forecasting" 2010 Chinese Control and Decision Conference

[17] T. Padmapriya and V. Saminadan, "Inter-cell Load Balancing technique for multi-class traffic in MIMO-LTE-A Networks", International Journal of Electrical, Electronics and Data Communication (IJEEDC), ISSN: 2320- 2084, vol.3, no.8, pp. 22-26, Aug 2015

[18] S.V.Manikanthan and V.Rama"Optimal Performance of Key Predistribution Protocol In Wireless Sensor Networks" International Innovative Research Journal of Engineering and Technology ,ISSN NO: 2456-1983,Vol-2,Issue-Special -March 2017. 